\title{
Slow-release artificial tear inserts in the treatment of dry eyes resulting from the oculomucocutaneous syndrome
}

\author{
P. WRIGHT' ${ }^{1}$ AND R. VOGEL ${ }^{2}$
}

From ${ }^{1}$ Moorfields Eye Hospital, City Road, London ECIV 2PD, and ${ }^{2}$ Merck Sharp and Dohme Ltd, Hoddesdon, Herts EN11 9BU

SUMmary Patients with dry eyes resulting from the practolol syndrome present a therapeutic challenge. Very frequent instillation of preservative-free drops are the only therapy in most cases. Using an entirely new agent - a hydrophilic polymer in insert form-we have provided in this open study an alternative therapy which shows some subjective and objective advantages. Few patients experienced problems, and none left the study because of failure of retention of the insert. Some patients preferred the cooling, soothing effects of frequent drops. The problems in mounting a clinical trial of this sort are discussed.

The use of practolol in the management of coronary insufficiency and hypertension in the early 1970 s resulted in the syndrome of skin rash, deafness, dry eyes, and pleural and peritoneal effusion and fibrosis (oculomucocutaneous syndrome) in some patients taking the drug. ${ }^{1}$ The ocular involvement, although variable in extent, was associated with reduction in tear production, reduction in lysozyme content of the tear film, and conjunctival fibrosis. The persistent and troublesome nature of the dry eyes experienced by some of these patients has resulted in their being obliged to use tear substitutes constantly. In many cases drops have to be instilled every 15-20 minutes throughout the day and occasionally during the night.

Many different agents and systems have been tried in the management of the extremely dry eye and the OMC syndrome is probably the severest form of this condition. So far no substitutes have been found for frequent artificial tears-whether purely aqueous replacement as in physiological saline drops or aqueous and wetting agents such as in gutt. hypromellose or gutt. alk. Since the introduction in $1945^{2}$ of methylcellulose into ophthalmology a number of other mucin replacements have followed. These include hydroxypropyl methylcellulose, carboxycellulose, and more recently polyvinyl alcohol. Efforts to demonstrate the superiority of one type of tear substitute over another have led to conflicting results. No study has satisfactorily shown that the

Correspondence to Dr R. Vogel. solutions remain in contact with the cornea longer than 15 min after instillation. ${ }^{3}$ An added problem of which clinicians have become increasingly aware is the reactions to the various preservatives necessary in these solutions.

In an attempt to overcome these problems Katz and Blackman ${ }^{4}$ and Lamberts et al. ${ }^{5}$ have used a slow-release artificial tear insert (Merck Sharp and Dohme) with some success. The insert is a $5 \mathrm{mg}$ rod of hydroxypropyl cellulose (molecular weight $c$

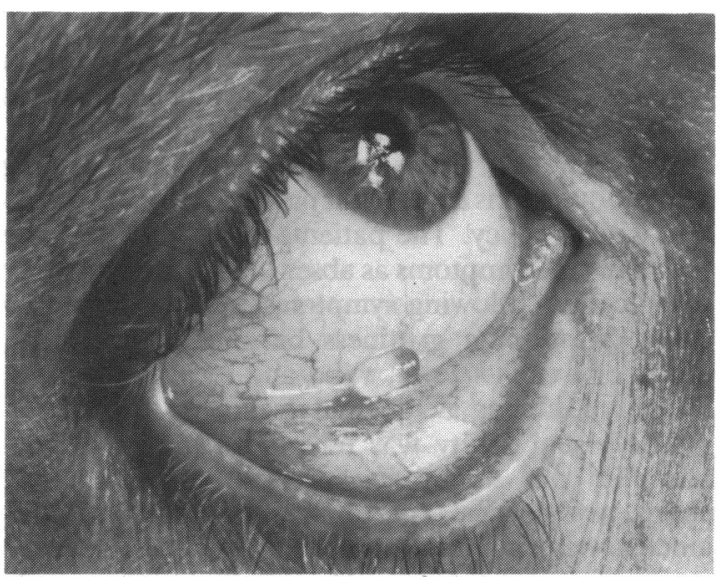

Fig. 1 Slow release artificial tear insert in lower fornix. The insert is initially a rod which swells and forms an ovoid as shown here. 
1000000 ) measuring approximately $3 \times 1 \mathrm{~mm}$. It is inserted into the inferior fornix, where it imbibes water from the conjunctiva. Within a few minutes the insert swells to form a small soft mass (Fig. 1), dissolving to coat the conjunctiva and cornea with a hydrophilic film which stabilises the tear film and hydrates and lubricates the cornea. Day-long relief of dry eye symptoms has been reported ${ }^{5}$ from a single insert placed in the eye early in the morning. Since these inserts do not need to contain any preservatives, the problems inherent in prolonged exposure to this group of troublesome agents should be avoided.

Since many patients with OMC syndrome have experienced the need to use tear replacement therapy so frequently and even then with incomplete relief, we determined to evaluate the inserts in this well documented group of patients.

\section{Patients and methods}

Patients were recruited from the OMC syndrome records of Moorfields Eye Hospital, City Road, London, if their last clinic note showed them to be requiring artificial tears at least every 2 hours. The following were exclusions to entering the study: (1) evidence of infection of conjunctiva or lids in the week preceding the study; (2) intraocular surgery or severe ocular trauma within 2 months; (3) deformity of the conjunctival sac sufficient to preclude retention of the insert; (4) abnormality of lid position or movement (entropion, ectropion, lagophthalmos, trichiasis, etc.); (5) patient currently wearing contact lenses or having done so during the past 2 months; (6) history of dendritic keratitis; (7) history of retinal detachment, diabetic retinopathy, or any progressive retinal disease; (8) ocular treatments other than artificial tears or acetylcysteine within 24 hours prior to onset of study.

The study was an open clinical study in which patients were offered the inserts as an alternative to their existing drops. None declined to enter the study.

On their first visit a history was taken including specific questions about their past and present therapy and its frequency. The patients were asked to score their current symptoms as absent, mild, moderate, or severe. The following symptoms were evaluated by direct questioning: grittiness, burning, photophobia, effect of irritants (e.g., wind and smoke), dryness, discharge, itching, aching, lid swelling.

The visual acuity was recorded. The precorneal tear film was scored for marginal strip thickness, debris, and mucus. The bulbar and palpebral conjunctiva was scored for hyperaemic follicles, papillae etc. and fornix shortening. The cornea was examined, and any abnormalities were noted and scored.

The eyes were then stained with rose Bengal 1\%, the excess being irrigated away with physiologicat saline, after which the staining of the cornea an conjunctiva was recorded.

The patients were then instructed in the insertion of the inserts with the applicator provided. They we told to insert one each morning in each eye, to use their usual tear substitutes if their symptonus warranted it, and that they could use as many inser: during the day as they found necessary for comfort. $\frac{\vec{\sigma}}{\vec{\sigma}}$

They were seen a week later (week 1), 2 weeks after that (week 3 ), and then finally (week 5). The eyes were fully examined as described above at eaci visit, but the symptom check was completed only on the final visit.

\section{Results}

A total of 11 patients entered the analysis, of whom 3 were male and 8 female. Their average age was 64 e् years. Five patients completed the protocol.

At the prestudy examination all the patients bu one were using tear substitutes more frequently than every half hour. Four patients were using alkalire drops, the remainder were using physiological saline drops without preservative. In addition 6 patier were also using gutt. acetylcysteine of various cofr centrations, and 2 were using gutt. predsol. Punct鸟 occlusion had been carried out in every case.

All the patients had some evidence of conjunctivత্త్(t fibrosis, but none had more than moderate forn shortening. Most patients were taking concurre therapy for their cardiovascular insufficiency and related problems. None of these are currently know to affect tear physiology adversely. All stopped taking practolol in 1974-5, and the average period for which practolol was taken was 3 years.

All observations and symptoms were scored at 0.

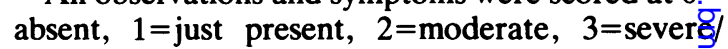
considerable. The rose Bengal staining was charted att the time of examination but was not scored until the study was completed, at which time all the records were scored at one session. The results expressed as mean scores for the prestudy examination and fin (week 5) examination are shown in Table 1. Table 1 Mean symptom scores prestudy and at 5 weeks

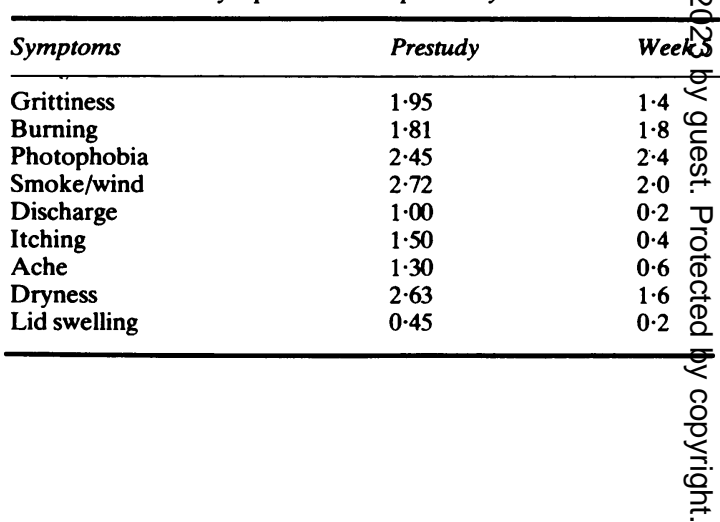




\begin{tabular}{|c|c|c|c|c|}
\hline SYMPTOM SCORR. & (absent) & $\begin{array}{c}1 \\
\text { slight }\end{array}$ & $\stackrel{2}{2}$ & $\begin{array}{c}3 \\
\text { Severe }\end{array}$ \\
\hline Grittiness & $\begin{array}{l}0 \\
0\end{array}$ & & 8 & 8 \\
\hline Burning & & O & 8 & 8 \\
\hline Photophobia & & - & 8 & ○ \\
\hline Smoke etc. & & 8 & 0 & 8 \\
\hline Discharge & $\begin{array}{rr} & 0 \\
8 & 0 \\
& 0 \\
\end{array}$ & 8 & $\bullet$ & \\
\hline Itching & - & 8 & & 8 \\
\hline Ache & 8 & 0 & 은 & - \\
\hline Dryness & 0 & 8 & $\bullet$ & 8 \\
\hline Lid Swelling & $8 \quad 80$ & 요 & & \\
\hline $\begin{array}{l}\text { Totals } \\
\text { Patient s }\end{array}$ & $\begin{array}{l}\bullet=16 \\
\text { absent or } s\end{array}$ & $\begin{array}{l}=29 \\
1 \mathrm{ight}\end{array}$ & $\begin{array}{l}\boldsymbol{\theta} \\
=29 \\
\text { madera }\end{array}$ & $\begin{array}{l}O=16 \\
\text { te or severe }\end{array}$ \\
\hline
\end{tabular}

Fig. 2 Symptom scores prestudy and at 5 weeks. $=$ One patient's score prestudy. $\bigcirc=$ One patient's score after 5 weeks of slow-release artificial tear inserts.

\section{Mean symptom scores at prestudy and week 5 visits}

In Fig. 2 is shown the number of patients who completed the protocol, each score being given for the prestudy examination and at week 5 . At the foot of this Figure can be seen the total patients scoring 0 or 1 (i.e., symptom absent or very slight) compared with those scoring 2 or 3 (i.e., symptom moderate or very troublesome). Whereas prior to using the dry eye inserts the score total in the absent or slight group was 16 , it had risen to 29 after 5 weeks on the inserts. Those scoring moderate or severe symptoms diminished correspondingly. Fig. 3 shows the scoring for precorneal tear film and marginal strip and Fig. 4 shows the scoring for rose Bengal staining.

All the patients entering the study had some degree of fornix shortening due to the fibrosis of the OMC syndrome. All except one patient experienced some difficulty from ejection of the inserts, but all found a means of overcoming this, and no patients left the study purely because they were unable to retain the inserts.

All those patients who stopped using inserts before the end of the protocol reported that their drops gave more instant relief than did the inserts. Three patients reported a greater cooling and soothing effect from instillation of drops than they got from the inserts. Two patients left the study because they were unable to tolerate the inserts. One patient reported an increase in frequency of instillation of drops one week after starting using the inserts and was withdrawn.

No local or systemic adverse reaction resulted from the use of up to 5 dry-eye inserts per day in the patients studied. There was no change in the slit-lamp appearance of the inferior fornix. The visual acuity remained unaltered on average during the study.

\section{Discussion}

We chose this group of patients for this, the first, trial of the dry-eye inserts in the UK mainly because they were so severely handicapped and because they were

\begin{tabular}{|c|c|c|c|c|c|c|c|}
\hline SCORE & $\begin{array}{c}0 \\
\text { (absent) }\end{array}$ & 1 & 2 & 3 & & & \\
\hline Marginal strip & - & $\because$ & 8 & 0 & $\frac{0-1}{e=8}$ & TOTAL & $\begin{array}{l}2-3 \\
0=2\end{array}$ \\
\hline Film thickness & & 8 & $\begin{array}{r}0 \\
-\quad 8 \\
\end{array}$ & & $0=2$ & & $O=8$ \\
\hline Debris & & $\bullet$ & $: \quad 80$ & & $\frac{0-1}{0=4}$ & TOTAL & $\begin{array}{l}2-3 \\
-=6\end{array}$ \\
\hline Mucus & 0 & $\begin{array}{ll}0 & 8 \\
- & 0\end{array}$ & : & & $0=6$ & & $0=4$ \\
\hline
\end{tabular}

Fig. 3 Precorneal tear film scores. $=$ One patient's score prestudy. $\bigcirc=$ One patient's score after 5 weeks of slow-release artificial tear inserts. 


\begin{tabular}{|c|c|c|c|c|}
\hline SCORE & 0 & 1 & 2 & 3 \\
\hline Cornea & & - & 8 & 8 \\
\hline Con j. & 0 & $\bullet$ & $:$ & : \\
\hline Totals & $=2$ & $O=\$$ & $\bullet=s$ & $O=2$ \\
\hline
\end{tabular}

Fig. 4 Corneal and conjunctival rose Bengal staining. $\mathbf{O}=$ One patient's score prestudy. $\mathrm{O}=$ One patient's score after 5 weeks of slow-release artificial tear inserts.

well documented. They were chosen for inclusion much as one would choose patients for any clinical treatment. It certainly became apparent that in the course of 7 or 8 years many of these patients had come to rely on their drops for cooling and soothing effects at least as much as for hydration and lubrication.

That only about half the patients completed the protocol is roughly in line with findings in contact lens tolerance and may be considered more a comment on the acceptability of inserting any solid object into the eye. Others have found a higher level of acceptance than we found, but their studies were with keratoconjunctivitis sicca. Breslin et al. ${ }^{7}$ reported that 4 out of 24 patients with keratoconjunctivitis sicca withdrew from their study because of symptoms related to the inserts. Two of their patients reported intolerable blurring of vision sufficient to stop them from using the inserts. Nobody in our series found this to be a problem, though many of our patients had a considerable degree of visual impairment (mean 6/24) before entering the study.

In a cross-over study comparing slow-release artificial tear inserts with liquid artificial tears Katz et al. ${ }^{6}$ found that $78 \%$ of their 32 keratoconjunctivitis sicca patients preferred inserts to drops, with only 5 patients out of a 47-patient long-term trial of inserts dropping out. One of their patients dropped out because of rheumatoid arthritis affecting his ability to insert the inserts and another because of bilateral aphakia. Others who dropped out did so because of blurring of vision due to the polymer in the tear film, although most who reported this-usually 4-6 hours after insertion-coped with it by removing the remaining portions of the insert without any diminution of the stabilising and lubricating effect.

Lamberts et al. ${ }^{5}$ reported that 18 of 37 patients in a long-term study of up to 18 months were happy to continue on the inserts. They found a significant increase in tear meniscus height after inserts compared with drops.

This study relies heavily on subjective criteria mainly because of the lack of reliably reproducible objective criteria when measuring tear films and wh recording patients' symptoms. Various parametê̈rs were considered for inclusion and discarded becamse we could not find sufficient evidence to support their reliability. Schirmer's test, which has long been used as a test for the dry eye, has many drawbacks, not he least being a poor correlation with symptomatology. The reliability of the tear break-up time (BUT) Fas been questioned, and Breslin et al. ${ }^{7}$ described graat difficulty in measuring it. We might with hindsig̈ht have used the measured depth of the marginal tor strip (meniscus) as used by Lamberts et al. ${ }^{5}$ The rese Bengal staining of the cornea and conjunctiva give hoped would prove an objective means of followe解 the epithelial surface during the study. In the ever it provided much useful information but was responsiBle for a considerable irritation and increase in local symptoms in the day or two following examinations, and symptoms from this cause had to be separated from any due to the inserts, particularly during the first week of use. A nonirritant vital stain to replace rose Bengal would be a great addition to the ophthalmic diagnostic armamentarium.

We were, however, convinced of the validity of $\overrightarrow{\text { gur }}$ scoring method which, although rather coarse, elid adequately reflect the subjective trend in the patiegrs. A significant improvement in marginal strip and tar film scores is related to the hydrophilic bulking effect of the polymer, and this in turn is reflected in the reduction in rose Bengal staining of the cornea $a_{\infty}$ It would have been acceptable objectively if these parameters had remained more or less the same during the study. Replacement of a 4 times an hour regimen by a once or twice daily regimen confer the same benefit would in itself be accepted.

In this extremely difficult group of patients we have found an encouraging level of acceptance of this treatment system. We have also demonstrăred objective and symptomatic improvement in those patients able to complete the study protocol. No serious adverse effects were reported or recorded It would be worth considering the use of these or simfitar inserts in the management of the troublesome tifyeye patient.

$$
\text { ก }
$$

We are grateful to ICI and Merck Sharp and Dohme Ltdiर्for supporting this study, and to Merck Sharp and Dohme for suppying the materials. Mrs S. Morris gave invaluable help with organisation and Miss Teresa Robinson with preparation of the manuscript. 6

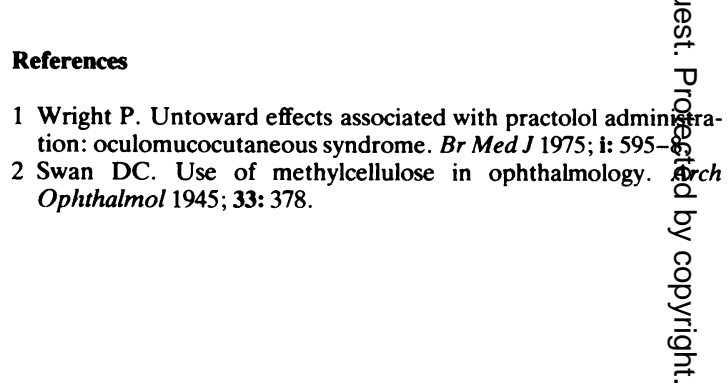


3 Lemp MA. Tear substitutes in the treatment of dry eyes. Int Ophthalmol Clin 1973; 13: 145-53.

4 Katz MK, Blackman WM. A soluble sustained-release ophthalmic delivery unit. Am J Ophthalmol 1977; 83: 728-34.

5 Lamberts DW, Langston DP, Chu W. A clinical study of slowreleasing artificial tears. Ophthalmology 1978; 85: 794-800.
6 Katz JI, Kaufman HE, Breslin C, Katz IM. Slow release artificial tears and the treatment of keratitis sicca. Ophthalmology 1978; 85: 787-93.

7 Breslin C, Katz JI, Kaufman HE, Katz IM. Slow release artificial tears. In: Leopold LH, Burns RP, eds. Symposium on ocular therapy. New York: Wiley, 1977; 10: 77-83. 